\title{
Efficacy of a novel chest tube system in a swine model of hemothorax
}

\author{
Ross I. Donaldson ${ }^{1,2,3,4}$, Eric M. Zimmermann ${ }^{5,6}$, Oliver J. Buchanan ${ }^{1}$, Todd L. Graham ${ }^{5}$, James D. Ross ${ }^{5,7}$ \\ ${ }^{1}$ Critical Innovations, Los Angeles, CA, USA; ${ }^{2}$ Department of Emergency Medicine, David Geffen School of Medicine at UCLA, Los Angeles, CA, \\ USA; ${ }^{3}$ Department of Emergency Medicine, Harbor-UCLA Medical Center, Torrance, CA, USA; ${ }^{4}$ Department of Epidemiology, UCLA-Fielding \\ School of Public Health, Los Angeles, CA, USA; ${ }^{5}$ Department of Surgery, Oregon Health \& Science University School of Medicine, Portland, \\ OR, USA; ${ }^{6}$ Department of Surgery, New York Presbyterian Queens, New York City, NY, USA; ${ }^{7}$ Charles T Dotter Department of Interventional \\ Radiology, Oregon Health \& Science University, Portland, OR, USA \\ Contributions: (I) Conception and design: RI Donaldson, JD Ross; (II) Administrative support: OJ Buchanan, TL Graham; (III) Provision of study \\ materials or patients: RI Donaldson, OJ Buchanan; (IV) Collection and assembly of data: All authors; (V) Data analysis and interpretation: All \\ authors; (VI) Manuscript writing: All authors; (VII) Final approval of manuscript: All authors. \\ Correspondence to: James D. Ross, PhD. Associate Professor of Surgery, Division of Trauma, Critical Care \& Acute Care Surgery, Department of \\ Surgery, Oregon Health \& Science University, 3181 Southwest Sam Jackson Park Road, Portland, OR 97239, USA. Email: rosja@ohsu.edu.
}

\begin{abstract}
Background: Tube thoracostomy is the definitive treatment for most significant chest trauma, including injuries resulting in pneumothorax, hemothorax, and hemopneumothorax. However, traditional chest tubes fail to sufficiently remove blood up to $20 \%$ of the time (i.e., retained hemothorax), which can lead to empyema and fibrothorax, as well as significant morbidity and mortality. Here we describe the use of a novel chest tube system in a swine model of hemothorax.

Methods: This was an intra-animal-paired, randomized-controlled study of hemothorax evacuation using the PleuraPath ${ }^{\mathrm{TM}}$ Thoracostomy System (PPTS) compared to a traditional chest tube in large YorkshireLandrace swine $(75-85 \mathrm{~kg})$. One liter of autologous whole blood was infused into each pleural cavity simultaneously with subsequent drainage from each device individually monitored for a total of 120 minutes, before the end of the experiment and necroscopy.

Results: Six animals completed the full protocol. On average, the PPTS removed $17 \%$ more blood $(\mathrm{P}=0.049)$ and left $19.1 \%$ less residual hemothorax $(\mathrm{P}=0.023)$ as compared to the standard of care during the first two hours of use. No complications or iatrogenic injury were identified in any animal for either device.

Conclusions: The novel PPTS device was superior to the traditional chest tube drainage system in this acute, large-animal model of retained hemothorax. While this study supports clinical translation, further research will be required to assess efficacy and optimize device use in humans.
\end{abstract}

Keywords: Retained hemothorax; trauma; thoracostomy; torso; pneumothorax

Submitted Apr 08, 2020. Accepted for publication Sep 09, 2020.

doi: $10.21037 /$ jtd-20-1609

View this article at: http://dx.doi.org/10.21037/jtd-20-1609

\section{Introduction}

Tube thoracostomy is the definitive treatment for most significant trauma, including injuries resulting in pneumothorax, hemothorax, and hemopneumothorax (1). However, traditional chest tubes fail to sufficiently remove blood up to $20 \%$ of the time (i.e., retained hemothorax), which can lead to empyema and fibrothorax, as well as significant morbidity and mortality $(2,3)$.

One of the main drawbacks of the traditional chest tube is that the portion of the device outside the body immediately becomes unsterile after finishing the tube thoracostomy procedure. Therefore, if retained hemothorax is later identified (e.g., on chest X-ray or computed tomography), the chest tube cannot be easily moved to 
target the buildup (4). This same problem also occurs if the initial chest tube placement is too shallow or if it becomes dislodged or clogged. A survey of several hundred North American cardiothoracic surgeons and nurses found that clogging leading to chest tube dysfunction is a major concern among such providers, with all respondents (100\%) having observed this complication and the vast majority (87\%) reporting adverse patient outcomes from it (5). Over half $(51 \%)$ of surveyed surgeons additionally stated they were unsatisfied with currently available tube thoracostomy technology (5).

Under the current standard of care, retained hemothorax is primarily treated with early video-assisted thoracoscopic surgery (VATS) (6). An advantage of prompt intervention is that it reduces the risk of activating local fibrinolysis and thus risk of recurrent hemothorax (7). However, VATS requires specialized surgical skills, is expensive, takes up operating room time, necessitates general anesthesia with a double-lumen endotracheal tube, and is contraindicated in some patients (e.g., those with spinal injuries or marginal lung function). Thus, a method of preventing or removing retained hemothorax that precludes VATS would be clinically useful.

Several methods have been explored to prevent retained hemothorax. Variations in static chest tube positioning have been considered, but have failed to yield meaningful changes in rates of retained hemothorax (3). Two studies using a sterile Yankauer suction catheter to remove early clot resulted in conflicting data, with one study demonstrating a reduction in secondary interventions but the other failing to record a similar decrease $(8,9)$. Finally, a preliminary, nonrandomized pilot study of preventative thoracic irrigation with $1 \mathrm{~L}$ of warmed $0.9 \%$ saline upon initial chest tube placement demonstrated a reduced secondary intervention rate (3). However, to date a well-evidenced and widelyaccepted method for preventing retained hemothorax remains to be determined.

In this study, the investigators proposed to investigate the efficacy of a novel chest tube system [PleuraPath ${ }^{\mathrm{TM}}$ Thoracostomy System (PPTS), Critical Innovations LLC., Inglewood, CA, USA] to prevent retained hemothorax. We hypothesized that PPTS would be more effective than a traditional chest tube drainage system as determined by total volume of blood and weight of clot removed in a novel swine model of hemothorax. We present the following article in accordance with the ARRIVE reporting checklist (available at http://dx.doi.org/10.21037/jtd-20-1609).

\section{Methods}

\section{Regulatory approval and animal preparation}

This study took place at Oregon Health and Science University (OHSU), an Association for Assessment and Accreditation of Laboratory Animal Care accredited facility. OHSU's Institutional Animal Care and Use Committee, as well as the U.S. Army's Animal Care and Use Review Office, reviewed and approved this protocol prior to initiation of the experiment. Experiments were performed under Protocol Number IP00002521 granted by OHSU's Institutional Animal Care and Use Committee in compliance with institutional guidelines for the care and use of animals.

Swine (Sus scrofa, $75-85 \mathrm{~kg}$ ) from a single source vendor (Oak Hill Genetics) were sedated with $6-8 \mathrm{mg} / \mathrm{kg}$ of telazol and $0.005 \mathrm{mg} / \mathrm{kg}$ of glycopyrrolate. Animals were induced via face mask with isoflurane and $100 \%$ oxygen, orotracheally intubated, and placed on mechanical ventilation with isoflurane administered at $1-3 \%$ to maintain a surgical plane of anesthesia. Mechanical ventilation was set on volume control with a tidal volume of $8 \mathrm{~mL} / \mathrm{kg}$ and the respiratory rate was adjusted to maintain an end tidal $\mathrm{CO}_{2}$ between 38 and $42 \mathrm{mmHg}$. Immediately following intubation, $0.005-0.01 \mathrm{mg} / \mathrm{kg}$ of buprenorphine was administered for analgesia. The animals remained supine on the operating table for the duration of the experiment. A Drager Infinity M540 monitor collected physiologic data (e.g., temperature, blood pressure, heart rate) and a Perseus A500 monitoring device recorded ventilator data [e.g., endtidal $\mathrm{CO}_{2}$, airway pressure $\left(\mathrm{P}_{\text {aw }}\right)$, tidal volume] at 1-minute intervals (Drager Gateway Medical Cockpit Software).

The right external jugular vein was catheterized to provide access for administration of fluids, as well as the bilateral carotid arteries to provide access for phlebotomy and continuous invasive blood pressure monitoring (preferably using the right side). Finally, thoracostomy "procedure areas" were marked bilaterally at the 4th intercostal space, midaxillary line by a combination of anatomic landmarks and palpation.

\section{Randomization and device insertion}

Device side was randomized for each animal and then bilateral mini-thoracotomies were performed via the classic open approach through the previously marked areas with tubes placed superior-posteriorly at a depth of $8-10 \mathrm{~cm}$. For 


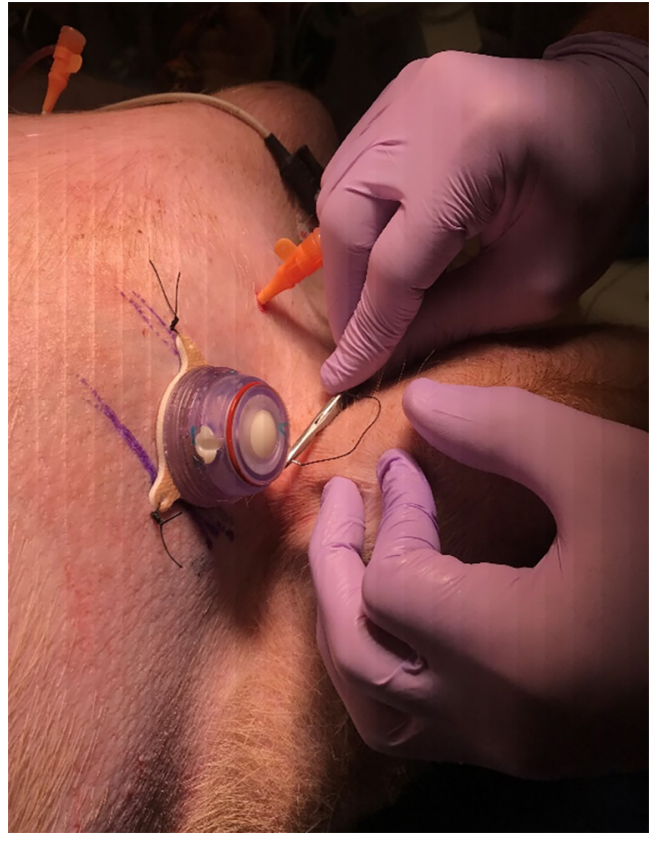

Figure 1 Securing PPTS port after insertion (prior to attachment connection). PPTS, PleuraPath ${ }^{\mathrm{TM}}$ Thoracostomy System.

the side with control device insertion, a traditional 36-Fr chest tube (Teleflex, USA) was used and secured in standard fashion (i.e., shoelace suture, occlusive gauze, gauze, tape). For the PPTS intervention side, a PPTS port with chest tube attachment (36 Fr) was placed and secured via the device's adhesive backing and suture-eyelets (Figure 1). Both the traditional and PPTS chest tubes were then connected to individual dry-suction suction apparatuses (Ocean 2050, Atrium), with the suction off and tubes clamped to prevent drainage (Figure 2).

\section{Creation of retained bemothorax}

Bilateral thoracic access was then additionally obtained using the Seldinger technique and 7-Fr introduction catheters in the $3^{\text {rd }}$ intercostal spaces at the anterior-axillary line and their position in the thoracic cavity confirmed via air aspiration. Subsequent to catheter placement, $1 \mathrm{~L}$ of native blood was withdrawn simultaneously from both carotid artery lines and infused into their respective pleural cavities at a rate of $50 \mathrm{~mL}$ per minute per side, for a total of $2 \mathrm{~L}$ bilaterally over 20 minutes. After 15 minutes of controlled hemorrhage and induction of hemothorax, $3 \mathrm{~L}$ of $5 \%$ Dextrose in Lactated Ringer's (Baxter, Deerfield, IL, USA) was given via bolus to prevent cardiovascular collapse.

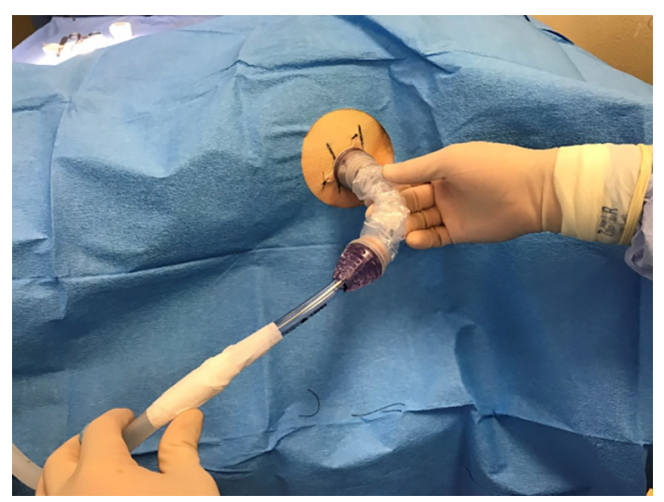

Figure 2 PPTS chest tube inserted into a swine and connected to suction. PPTS, PleuraPath ${ }^{\mathrm{TM}}$ Thoracostomy System.

\section{Retained hemothorax evacuation}

Thirty minutes later, to initiate hemothorax evacuation, clamps were simultaneously released from both the traditional and PPTS chest tubes with placement to $20 \mathrm{cmH}_{2} \mathrm{O}$ of continuous negative pressure (time $=0$ ). In the traditional chest tube group, the tube was not milked, tapped, stripped, or otherwise manipulated. In the PPTS group, the system was used per manufacturer's instructions to move the device's chest tube, within its outer sheath that maintains sterility as a function of the device, around the pleural cavity for 10 minutes using a "vacuuming" motion to remove clot. This included inserting and withdrawing the tube and rotating it to reach anterior/posterior and inferior/ superior positions. If a chest tube attachment became fully clotted, protocol allowed it to be exchanged for one or more additional chest tube attachments without removal of the inserted port.

After completion of this initial vacuuming period (time $=10$ ), both devices were left to suction for a total of 120 minutes (time=120). The traditional chest tube was never manipulated. For the PPTS group, the "vacuuming" procedure was repeated a second time for an additional 10 minutes starting at the midpoint of the experiment (time=60).

\section{Additional data collection and pathology}

Collected blood from each device was recorded at 10-minute intervals for the full 120-minute study period. At the end of this period, the animals were euthanized with $1 \mathrm{~mL} / 10 \mathrm{lb}$ of euthanasia solution. A median sternotomy was then performed and the thoracic cavity explored to 
Table 1 Study animal characteristics and necropsy findings

\begin{tabular}{lcccccc}
\hline Animal ID & Weight $(\mathrm{kg})$ & PPTS insertion site & Cardiac arrythmia & Cardiac injury & Pulmonary injury & Diaphragm injury \\
\hline 1 & 80 & Left & No & No & No & No \\
2 & 78 & Right & No & No & No & No \\
3 & 79 & Left & No & No & No & No \\
4 & 85 & Right & No & No & No \\
5 & 79 & Right & No & No & No & No \\
\hline
\end{tabular}

PPTS, PleuraPath TM Thoracostomy System.

Table 2 Study results

\begin{tabular}{lcccc}
\hline Data point & PleuraPath $^{\text {TM }}[\mathrm{SD}]$ & Standard [SD] & Absolute difference Relative difference & $P$ value \\
\hline $\mathrm{T}=10$ drainage $(\mathrm{mL})$ & $509[85]$ & $252[139]$ & 257 & $202 \%$ \\
$\mathrm{~T}=120$ drainage $(\mathrm{mL})$ & $695[65]$ & $592[57]$ & 103 & $117 \%$ \\
Residual hemothorax $(\mathrm{g})$ & $343[39]$ & $424[63]$ & -81 & $-19.1 \%$ \\
\hline
\end{tabular}

${ }^{*}$, statistically significant $(\mathrm{P}<0.05)$. Drainage was analyzed using two-way repeated measures ANOVA. SD, standard deviation.

confirm correct placement of both devices inside their respective pleural cavities. Residual blood and clots in each pleural space were then removed and carefully weighed to record residual volume. Cardiac, pulmonary and diaphragmatic injury was grossly assessed in a standardized, non-blinded fashion by the research team. If any team member felt that there was evidence of an injury, then it was recorded.

\section{Statistical analysis}

Experimental group size (n) was determined by using an $a$ priori power analysis $\left(\mathrm{G}^{*}\right.$ Power 3.1.9.7). The investigators made the assumption that a $150.0-\mathrm{mL}$ difference in chest tube output would be experimentally and clinically relevant. Additionally, assuming a predicted first standard deviation of $75.0 \mathrm{~mL}$ an effect size (d) of 1.7 was calculated. With $\alpha=0.05$ and $1-\beta=0.8$ an $n$ of 6 animals per group was determined. The actual a priori power calculation for this study was 0.86 for a total sample size of 12 (6 per group).

Residual hemothorax was analyzed with Student's $t$-test. Drainage data were analyzed with two-way repeated measures of ANOVA with post-hoc pairwise comparisons where appropriate (Holm-Sidak). All statistical analysis was performed using SigmaPlot 12 (SyStat Software Inc.).

\section{Results}

Of the nine swine, three were excluded from data analysis (two died before the intervention period and one due to the traditional chest tube placed incorrectly and found to be in the extra-thoracic space during necropsy), leaving a total of six animals $(n=6)$. These animals had an average weight of $80 \pm 2 \mathrm{~kg}$ and devices were randomized to the left and right sides equally (Table 1). Cardiac arrythmia was not observed in any animals, nor was there evidence of cardiac, pulmonary, or diaphragmatic injury on necropsy.

The primary endpoints for this study were defined as the paired difference in the amount of drainage from the PPTS and traditional devices at the end of the initial 10-minute and subsequent 120-minute periods. Analysis of these endpoints showed a statistically significant difference $(\mathrm{P}<0.05)$ between groups in drainage at 10 and 120 minutes, as well as residual hemothorax (Table 2). Overall, the difference was largest at the first timepoint (time=10), with the PPTS device draining more than twice as much blood on average. This advantage persisted, although at a lessened rate, out to 120 minutes, at which time the PPTS device had on average a $17-19 \%$ improvement over the current standard of care (as measured by drainage and residual hemothorax, respectively). 


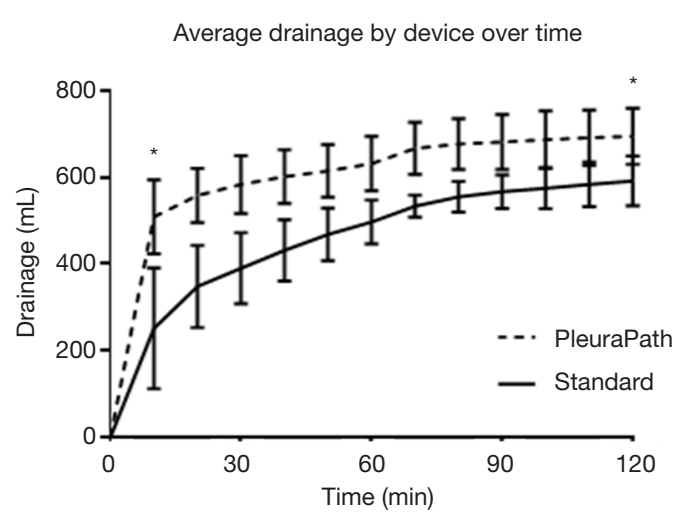

Figure 3 Average drainage by device over time. Analysis of time $=10$ and time $=120 \mathrm{~min}$ data points was determined $a$ priori, so no other time points were statistically analyzed.

\section{Discussion}

\section{Model development}

This study describes a novel model of retained hemothorax in adult-human-sized swine. It provides the benefit of producing paired data from bilateral contemporaneous interventions utilizing the animal's own blood without the use of any anticoagulants for storage or reversal agents to induce clot, as would be required if using extracorporal blood (autologous or homologous). The model expeditiously induced a sturdy clot in the pleural space via normal physiology. The production of paired data, with each animal acting as its own control, additionally strengthened statistical analysis by eliminating much of the potential for confounding variables between control and experimental groups.

In developing the model, we purposefully delayed the infusion of replacement intravenous fluids until 5 minutes prior to the completion of phlebotomy-hemothorax to minimize its effect on clotting via hemodilution. However, this likely resulted in a higher mortality rate than if the systemic infusion was commenced earlier in the hemorrhage protocol. A future model might use timelier fluid administration or performance of phlebotomy at a slower rate to allow better physiologic compensation. Alternatively, donor fresh whole blood could be sourced, however, donor blood would be stored in citrate and the reversal of calcium chelation prior to or subsequent to creation of the simulated hemothorax injury might add an unnecessary complication to the model.

\section{Device performance}

This intra-animal-paired, randomized-controlled evaluation of hemothorax evacuation using the PPTS demonstrated improved chest tube output as compared to the standard of care during the 2 hours of simulated use $(\mathrm{P}<0.05)$. The difference was greatest at the first sampling timepoint (with almost twice as much drainage), as measured immediately after completion of the "vacuuming" procedure performed with the PPTS device. This result was not unexpected and is likely attributable to the fact that the PPTS device can be manipulated in the pleural cavity via its protective outer sheath while maintaining sterility, while the traditional chest tube cannot be similarly repositioned.

This vacuuming procedure is similar to that previously described in humans utilizing a sterile Yankauer suction catheter to remove early clot $(8,9)$. However, the PPTS has the additional capability of repeated vacuuming sweeps, in addition to that performed upon initial insertion. As predicted by investigators, a second, although smaller, increase in drainage was measured in PPTS devices just after the second vacuuming procedure (time $=60$ ), as seen in Figure 3. In this study, protocol only allowed for performance of the vacuuming procedure twice, at set time points of 10-minute duration, although in a practical clinical setting a provider could perform it as many times as deemed necessary. Additionally, experimental protocol did not utilize complementary imaging to specifically identify the location of buildup, as would be reasonable and easily applied in human clinical use.

Although the study protocol allowed for replacement of a clotted PPTS chest tube attachment if necessary, which could be performed without need for a new sterile setup of the device, there were no episodes of clotting requiring a re-deployment procedure for any animal. This is likely due to the relatively short study duration (2 hours), with clotting more likely to occur over a period of days. Thus, the potential benefit of re-deployment was not tested in comparison to standard care in this study; doing so would likely require a longer duration study in a similar model with critical care protocols.

Overall, the PPTS demonstrated a statistically significant $17-19 \%$ relative improvement in drainage over standard care when measured 2 hours after placement (Table 2). In absolute terms, this was a reduction in 81 grams of residual hemothorax from the 1 liter of blood placed in a single the pleural cavity. This amount would be expected to be 
greater with larger or continuous bleeds and reduced for smaller hemothoraces. Although there is little data to guide judgement, the authors would suggest that this result appears to be a clinically significant reduction in retained hemothorax burden in this model and therefore relevant to the translated clinical experience.

The PPTS is a new technology and therefore there are multiple potential areas of interest for additional research. Future studies could additionally explore the combination of this device with warmed saline as described by Kugler et al. (3) or with intrapleural thrombolytics as described in other studies. Other possibilities of interest include utilizing different vacuuming protocols or their integration with imaging (e.g., X-ray, computerized tomography, point-ofcare ultrasound), possibly over a longer time duration.

\section{Conclusions}

We conclude from this study that a novel chest tube system that allows for continuous manipulation while maintaining sterility of the chest tube has the potential to provide significant benefit to patients requiring the evacuation of hemothoraces and retained clot. While the data presented herein is optimistic for successful clinical translation, further study will be required to assess human efficacy and to refine the use process.

\section{Acknowledgments}

Funding: This work was supported by the US Army Medical Research and Materiel Command under Contract No. W81XWH-17-C-0211.

\section{Footnote}

Reporting Checklist: The authors have completed the ARRIVE reporting checklist. Available at http://dx.doi. org/10.21037/jtd-20-1609

Data Sharing Statement: Available at http://dx.doi. org/10.21037/jtd-20-1609

Conflicts of Interest: All authors have completed the ICMJE uniform disclosure form (available at http:// dx.doi.org/10.21037/jtd-20-1609). Dr. RID reports other funding from US Army Medical Research and Materiel Command (Contract No. W81XWH-17-C-0211) and
Critical Innovations LLC., during the conduct of the study; other relevant financial activities from Critical Innovations LLC., outside the submitted work; In addition, Dr. RID has patents US 10,046,147 B2; 61/920,963; 14/581,339; 16/015,586; $14200292.262 / 562,183 ; 16 / 113,707$; 18195962.8 issued and pending. Mr. OJB reports other from US Army Medical Research and Materiel Command (Contract No. W81XWH-17-C-0211, other from Critical Innovations, LLC., during the conduct of the study; other from Critical Innovations, LLC., outside the submitted work; In addition, Mr. Buchanan has a patent 16/113,707 issued. Dr. TLG reports grants from US Army Medical Research and Materiel Command (Contract No. W81XWH-17-C-0211, outside the submitted work; Dr. JDR reports grants from US Army Medical Research and Materiel Command (Contract No. W81XWH-17-C-0211, during the conduct of the study. Dr. EMZ has no conflicts of interest to declare.

Ethical Statement: The authors are accountable for all aspects of the work in ensuring that questions related to the accuracy or integrity of any part of the work are appropriately investigated and resolved. Experiments were performed under Protocol Number IP00002521 granted by Oregon Health and Science University's Institutional Animal Care and Use Committee in compliance with institutional guidelines for the care and use of animals.

Disclaimer: The views, opinions and/or findings contained in this report are those of the author(s) and should not be construed as an official Department of the Army position, policy or decision unless so designated by other documentation. In conducting research using animals, the investigators adhered to the Animal Welfare Act Regulations and other Federal statutes relating to animals and experiments involving animals and the principles set forth in the current version of the Guide for Care and Use of Laboratory Animals, National Research Council.

Open Access Statement: This is an Open Access article distributed in accordance with the Creative Commons Attribution-NonCommercial-NoDerivs 4.0 International License (CC BY-NC-ND 4.0), which permits the noncommercial replication and distribution of the article with the strict proviso that no changes or edits are made and the original work is properly cited (including links to both the formal publication through the relevant DOI and the license). 
See: https://creativecommons.org/licenses/by-nc-nd/4.0/.

\section{References}

1. Katlic M Cardiothoracic Surgery in the Elderly. New york: Springer, 2001.

2. Boersma WG, Stigt JA, Smit HJ. Treatment of haemothorax. Respir Med 2010;104:1583-7.

3. Kugler NW, Carver TW, Milia D, et al. Thoracic irrigation prevents retained hemothorax: A prospective propensity scored analysis. J Trauma Acute Care Surg 2017;83:1136-41.

4. DuBose J, Inaba K, Demetriades D, et al. Management of post-traumatic retained hemothorax: a prospective, observational, multicenter AAST study. J Trauma Acute Care Surg 2012;72:11-22; discussion 22-4; quiz 316.

5. Shalli S, Saeed D, Fukamachi K, et al. Chest tube selection in cardiac and thoracic surgery: a survey of chest tuberelated complications and their management. J Card Surg 2009;24:503-9.

6. Mowery NT, Gunter OL, Collier BR, et al. Practice management guidelines for management of hemothorax and occult pneumothorax. J Trauma 2011;70:510-8.

7. Yeung C, Dawson J, Gilbert S. Uniportal videoassisted thoracoscopy approach to the management of non-pulmonary diseases of the chest. J Thorac Dis 2019;11:S2062-8.

8. Ramanathan R, Wolfe LG, Duane TM. Initial suction evacuation of traumatic hemothoraces: a novel approach to decreasing chest tube duration and complications. Am Surg 2012;78:883-7.

9. Savage SA, Cibulas GA 2nd, Ward TA, et al. Suction evacuation of hemothorax: A prospective study. J Trauma Acute Care Surg 2016;81:58-62.

Cite this article as: Donaldson RI, Zimmermann EM, Buchanan OJ, Graham TL, Ross JD. Efficacy of a novel chest tube system in a swine model of hemothorax. J Thorac Dis 2021;13(1):213-219. doi: 10.21037/jtd-20-1609 